\title{
Decomposition Algorithm of Laser Reflecting Waveform Based on Particle Swarm Optimization
}

\author{
Can Dai ${ }^{1, a,}$ Yuanqing Wang ${ }^{\star^{1, b}}$, Fan $\mathrm{Xu}^{1, \mathrm{c}}$ \\ ${ }^{1}$ School of Electronic Science and Engineering, Nanjing University, Xianlin Road 163,Xixia \\ District,Nanjing,210046,China

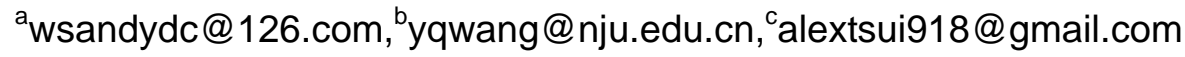

Keywords: LiDAR; Signal processing; Waveform decomposition; Particle Swarm Optimization

\begin{abstract}
The laser system's parameter fitting is an important step to the ranging and imaging, however, the complexity of the target object will cause the waveform superimposed, some existing algorithms are difficult to accurately obtain the parameters, by decomposing waveform into a series of continuous waveform can greatly improve the fitting accuracy. Considering the existing algorithm, LM algorithm's accuracy depends on the initial value and is easy to be caught in local optima, if the initial value is inaccurate then it is difficult to get an accurate fitting results. The laser we use is not the standard Gaussian model in reality,it is asymmetric,so if we directly use the value acquired by the Gaussian model,the result will be inaccurate. This paper will consider and propose a lidar waveform decomposition process based on the combination of the PSO algorithm and the LM algorithm in order to improve the final precision.
\end{abstract}

\section{Introduction}

After decades of research and development,three-dimensional laser imaging technology has been applied in many areas of remote sensing and measurement.This imaging technology has the advantages such as high precision,large measuring density,rich information and so on.It is easy to separate the features and the plants,and describes them with mathematical model[1,2].Now,this technology is progressively replacing the traditional stereoscopic photography,becoming the major technology to generate the Digital Surface Model (DSM) and Digital Terrain Model (DTM)[3].

The laser echo waveform contains lots of information of the target object[4].Many scholars have done great jobs on the decomposition.Hofton proposes the decomposition based on the Gaussian model[5].He considers the waveform as a sum of a series of Gaussian components.Zwally and Wagner point that Gaussian decomposition fits most of the industrial applications[6,7].

Now most of the decomposition algorithms are based on the Gaussian model,consider the waveform as the sum of the Gaussian components[8]. They use the data of peak points and symmetrical inflection points detected to define the initial values such as the delay time and pulse width.However,the laser model in our system is not completely Gaussian model and it is not symmetrical.The real waveform we use is shown in fig1.1.

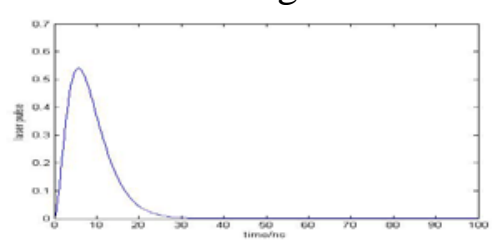

Fig1.1 the actual waveform we use

If we directly use the location value and amplitude value,the result will be inaccurate.The LM algorithm's accuracy relies on the initial values and it is easy to get in local minimum.Different initial values will lead to different results.So in this paper we propose a new algorithm which combines the Particle Swarm Optimization(PSO) algorithm with LM algorithm to get the global optimum and improve the final result. 


\section{Decomposition of Laser Reflecting Waveform}

\section{1 denoising and smoothing}

Background noise can cause the random change of waveform amplitude,too much burr will lead to the detection of the fault points.Some smoothing algorithms can cause the result to lack fidelity of the amplitude,at the same time,over smoothing will make the details lost or even affect the result of the detection of peak points.So it is necessary to choose the best one.In this paper,we choose the vondrak smoothing method.The vondrak method can not only used for equally spaced and precision data,but also for unequal spaced and precision data,so it has a wide range of applications. The Vondrak smoothing's basic assumption is:

$$
\begin{aligned}
\mathrm{Q} & =F+\lambda^{2} S=\text { Minimum } \\
F & =\sum_{i=1}^{n} P_{i}\left(\mathrm{y}_{i}-\bar{y}_{i}\right)^{2} \\
S & =\int_{x_{1}}^{x_{n}}\left[y_{i}\right]^{\prime \prime \prime}
\end{aligned}
$$

$\bar{y}_{i}$ is the data after the smoothing processing, ${ }^{y_{i}^{\prime \prime \prime}}$ represents the third derivative of $y_{i}, P_{i}$ represents the right of the measured data, $\lambda$ is the coefficient to be determined, $\mathrm{F}$ is called the approximation degree, $\mathrm{S}$ is named as the smoothing degree.

\subsection{Threshold setting and peak \& inflection detecting}

This paper determines the number of waveform by detecting the number of peak points and inflections, in this way,we can divide the data into several separate waveform.However,even though we remove most of the noise and burr through denoising and smoothing,many wrong points will be detected because of the remaining noise.So it is necessary to set a threshold value due to the background noise.

In this paper,we will take a length of the data and consider it as the background noise.By calculating its average value and variance,we set the threshold as:

\subsection{Parameter fitting algorithm}

$$
\text { Thval }=\text { mean_value }+4 * \text { var_value }
$$

\subsubsection{Particle swarm optimization fitting}

The laser we use is not the real of Gaussian model,so the waveform is not symmetrical.Through detecting the peak point and inflection point,we get the number of the single waveform.Then we can get the parameters by using PSO algorithm.

The PSO algorithm is a parallel global searching algorithm based on swarm intelligence, using simple speed - position searching model to achieve the optimization operation in the whole space.The algorithm updates the particles by:

$$
V_{i m}^{k+1}=w^{*} V_{i m}^{k}+c_{1}^{*} \text { rand } *\left(\mathrm{P}_{i m}-x_{i m}^{k}\right)+c_{2}{ }^{*} \text { rand } *\left(\mathrm{P}_{g m}-x_{i m}^{k}\right)
$$

$V_{i m}^{k}$ means the flight speed of the $\mathrm{m} D$ of the ith of the kth generation particle,its max value is set as 0.5 ,the min value is set as $-0.5 . \mathrm{w}$ is the Inertia weight, ${ }^{{ }_{i m}^{k}}$ represents the best value of the ith particle found so far, ${ }^{k}{ }_{g m}^{k}$ represents the best value of the whole particle swarm found so far.

After the PSO algorithm fitting,the accuracy and precision can still be improved,so we combine it with LM algorithm.

\subsubsection{LM fitting}

Levenberg-Marquardt algorithm is the combination of the gradient descent and the Gauss Newton method,it provides a numerical solution to the problem of minimizing a function,generally nonlinear,over a space of parameters of the function.For well-behaved functions and reasonable starting parameters,the result will be very accurate.However,the LM algorithm is easy to be caught in a local minimum,so if it is combined with the PSO algorithm,the result will be improved.The LM algorithm's basic suppose is : 


$$
\begin{gathered}
F(\omega)=\frac{1}{2}\|\mathrm{r}(\omega)\|_{2}^{2} \\
\nabla F(\omega)=\mathrm{J}(\omega)^{T} r(\omega) \\
\nabla^{2} F(\omega)=\mathrm{J}(\omega)^{T} \mathrm{~J}(\omega)+\sum_{j=1}^{N} r_{j}(\omega) \nabla^{2} r_{j}(\omega)
\end{gathered}
$$

Among them, $\omega$ is the parameter to fit, $r(\omega)$ is the margin vector of the fitting data. $J(\omega)$ is the Jacobi matrix of $r(\omega)$.And the iteration is:

$$
\Delta \omega=-\left[\mathrm{J}^{T}(\omega) \mathrm{J}(\omega)+\mu \mathrm{I}\right]^{-1} J(\omega)^{T} r(\omega)
$$

\section{Data Processing and Validation}

Firstly,we get the data of the full-waveform lidar,it is shown in fig3.1(a).Then we deal it with denoising.The data after denoising is show in fig3.1(b).As we can see from it,the noise is clearly suppressed.

(a)

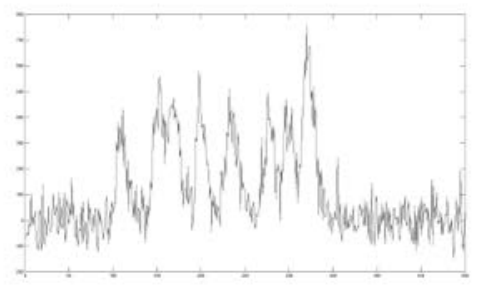

(b)

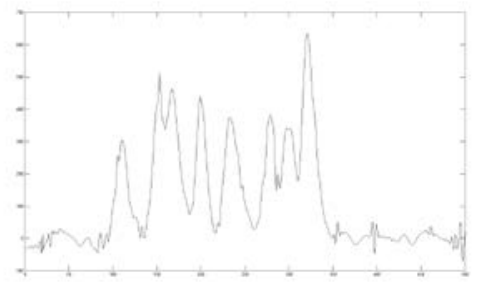

Fig3.1 the real signal and the denoised waveform

With denoising,burr still exist in it,so it is necessary to smooth it for ideal data.After the Vondrak smoothing,the result is described in fig3.2.

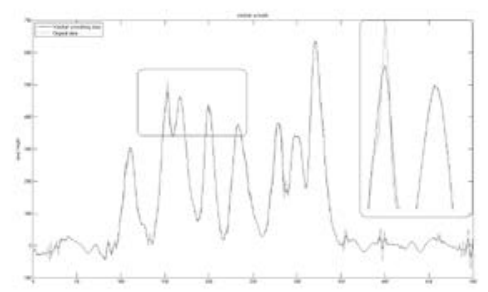

Fig3.2 the waveform after smoothing

Then we need to set a reasonable threshold value to determine the number of the waveform.As shown in the figure below,we take the front eighty points as background noise and then calculate the mean value and the variance.The threshold value is about 50 and it detects 8 single waveform.

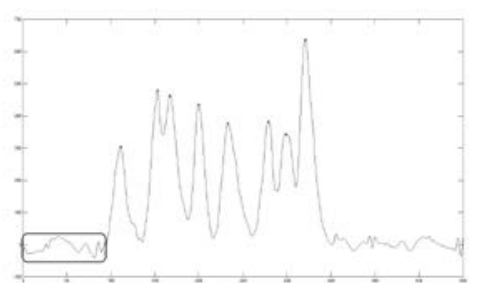

Fig3.3 get the background noise and set the threshold value

Firstly use particle swarm optimization algorithm to get a comparatively more accurate value to 
improve the final result.In the PSO algorithm,when the inertia factor uses 0.729 it can get a better convergence.Acceleration constant c1 and c2 in this paper we choose 1.454.For evaluating the final result conveniently,we propose the concept of the goodness in this paper,considering it:

$$
R=1-\sum_{i=1}^{N}\left(o b s_{i}-y_{i}\right)^{2} / \sum_{i=1}^{N} o b s_{i}^{2}
$$

The final result is shown in fig3.4.Compared with the data processed by only LM algorithm,the accuracy and precision is improved.The parameter is shown in table 1,and we can see the value we get through the PSO\&LM is more close to the real.The final $\mathrm{R}$ is 0.9892 .
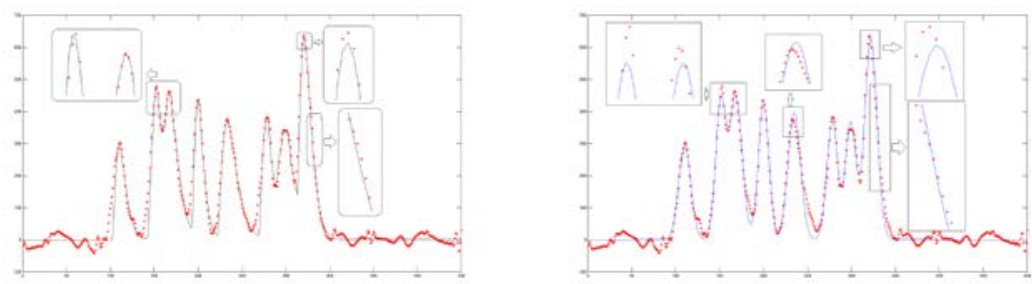

Fig3.4 the comparison of the result fitted by PSOLM and LM only

From the comparison we can see,the result using PSOLM gets a higher accuracy and precision than the one using LM algorithm only.Especially on the vertex's fitting,we can get a more accurate final result.

Table1 the parameter fitted by PSOLM and comparison with LM only

\begin{tabular}{c|c|c|c|c|c}
\hline No. & Width & $\begin{array}{l}\text { Amplitu } \\
\text { de }\end{array}$ & $\begin{array}{l}\text { Delay(PSOL } \\
\text { M) }\end{array}$ & $\begin{array}{c}\text { Real } \\
\text { delay }\end{array}$ & $\begin{array}{c}\text { Delay(L } \\
\text { M) }\end{array}$ \\
\hline 1 & $\begin{array}{c}13.475 \\
7\end{array}$ & $\begin{array}{c}507.972 \\
8\end{array}$ & 108.4511 & 108 & 110.4563 \\
\hline 2 & $\begin{array}{c}15.887 \\
6\end{array}$ & $\begin{array}{c}808.281 \\
9\end{array}$ & 151.5939 & 151 & 151.3806 \\
\hline 3 & $\begin{array}{c}17.711 \\
0\end{array}$ & $\begin{array}{c}764.235 \\
6\end{array}$ & 167.4733 & 167 & 168.6669 \\
\hline 4 & $\begin{array}{c}12.876 \\
5\end{array}$ & $\begin{array}{c}739.296 \\
3\end{array}$ & 206.8891 & 206 & 204.2180 \\
\hline 5 & $\begin{array}{c}15.790 \\
6\end{array}$ & $\begin{array}{c}649.027 \\
0\end{array}$ & 231.9724 & 231 & 235.0024 \\
\hline 6 & $\begin{array}{c}13.713 \\
3\end{array}$ & $\begin{array}{c}628.896 \\
2\end{array}$ & 277.7808 & 277 & 278.3579 \\
\hline 7 & $\begin{array}{c}12.156 \\
2\end{array}$ & $\begin{array}{c}532.870 \\
6\end{array}$ & 302.4133 & 302 & 299.2548 \\
\hline 8 & $\begin{array}{c}15.219 \\
7\end{array}$ & $\begin{array}{c}1062.34 \\
84\end{array}$ & 321.6134 & 321 & 322.1844 \\
\hline
\end{tabular}

For each wave decomposed ,its time delay value corresponds to the elevation information of the pixel,and its amplitude value corresponds to the intensity information of the pixel.

\section{Conclusion}

The decomposition algorithm of laser reflecting waveform in this paper gets each component's delay time,pulse width and amplitude information,and they represent the information of the location,intensity and so on.This algorithm can improve the accuracy and precision.However,each part will has a unignored effect on the final result.such as the number determined by the peak point\&inflection point detecting and so on.So for different target object,there should be a best fitting guideline.In practical applications,it still needs more data to verify the advantages of this algorithm. 


\section{Reference}

[1] LiDAR-Overview of Technology Applications Market Features \& Industry [R].Prepared by BC-CARMS,Centre for Applied Remote Sensing,Modelling and Simulation University of Victoria,Victoria,BC,2006

[2] Lei Li,Yihua Hu,et al.Application of Three-Dimensional Laser Imaging Technology[J].Laser\& Optoelectronics Progress(in Chinese),2009(12):66-70

[3] C Mallet,F Bretar.Full-waveform topographic lidar:State-of-the-art[J].ISPRS Journal of Photogrammetry and Remote Sensing,2009,64(1):1-16

[4] Xin Zhao,Yi Zhang,et al.Algorithm of laser altimeter reflecting waveform based on Gaussian decomposition[J].Infrared and Laser Engineering(in Chinese),2012(03):643-648

[5] Hofton M A,Minster J B,Blair J B. Decomposition of laser altimeter waveforms [J]. IEEE Transactions on Geoscience and Remote Sensing,2000,38(4):1989-1996.

[6] Zwally H,Schutz B,Abdalati W,et al. ICESat's laser measurements of polar ice atmosphere,ocean,and land[J].Photogrammetric Journal of Finland,2008,21(1):62-72.

[7] Wagner W,Ullrich A,Ducic V,et al. Gaussian decomposition and calibration of a novel small-footprint full-waveform digitising airborne laser scanner[J]. ISPRS Journal of Photogrammetry and Remote Sensing,2006,60(2):100-112

[8] Xudong Lai,Nannan Qin,et al.Iterative decomposition method for small foot-point LiDAR waveform[J].Journal of infrared and millimeter waves(in Chinese),2013,32(4):319-324 